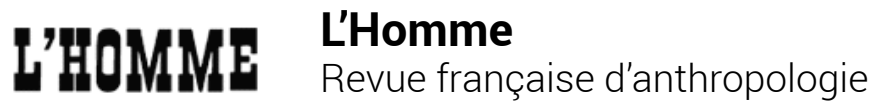

187-188| 2008

Miroirs transatlantiques

\section{Meera Kosambi, Crossing Threshholds. Feminist Essays in Social History}

Martine Van Woerkens

\section{(2) OpenEdition}

1 Journals

\section{Édition électronique}

URL : https://journals.openedition.org/lhomme/20542

DOI : 10.4000/lhomme.20542

ISSN : 1953-8103

Éditeur

Éditions de l'EHESS

\section{Édition imprimée}

Date de publication : 3 octobre 2008

Pagination : 471-475

ISBN : 978-2-7132-2186-6

ISSN : 0439-4216

\section{Référence électronique}

Martine Van Woerkens, "Meera Kosambi, Crossing Threshholds. Feminist Essays in Social History», L'Homme [En ligne], 187-188 | 2008, mis en ligne le 16 décembre 2008, consulté le 23 avril 2022. URL http://journals.openedition.org//homme/20542 ; DOI : https://doi.org/10.4000//homme.20542

Ce document a été généré automatiquement le 23 avril 2022.

(c) École des hautes études en sciences sociales 


\title{
Meera Kosambi, Crossing Threshholds. Feminist Essays in Social History
}

\author{
Martine Van Woerkens
}

\section{RÉFÉRENCE}

Meera KoSAMBI, Crossing Threshholds. Feminist Essays in Social History. Ranikhet, Permanent Black, 2007, 397 p., bibl., index, fig.

1 DANS LE CHAMP des études féministes indiennes, Meera Kosambi occupe une place éminente. Cette historienne qui dirigea de 1991 à 1998 le Centre de recherches des études sur les femmes à la SNDT Women's University à Mumbai ${ }^{1}$, s'est attachée depuis une vingtaine d'années à restituer les voix plurielles - oubliées ou « égratignées » par l'historiographie officielle - des femmes du Maharashtra au cours du XIX ${ }^{\mathrm{e}}$ siècle. Grâce à ses travaux, la réforme sociale et le nationalisme maharashtriens ne sont plus exclusivement le produit de la pensée et de l'action masculines. Cette époque tumultueuse se définit aussi par l'action et la réflexion des femmes. Crossing Threshholds donne la parole à six d'entre elles, sans cependant occulter ni caricaturer celle des hommes. En ce sens, l'ouvrage de Meera Kosambi renouvelle l'histoire sociale du Maharashtra car les acteurs enfin au complet sont traités avec la même équanimité.

2 Cette histoire sociale concerne essentiellement la fabrique intime de la société maharashtrienne des hautes castes, en d'autres termes, les coutumes et les règles qui fixaient les relations profondément asymétriques entre sexe et genre. Religieusement sanctionnées, ces coutumes et ces règles visaient à contrôler la sexualité féminine ${ }^{2}$. Le mariage précoce, l'« imprégnation » dès après les premières règles, à quoi s'ajoutait le tabou de l'éducation, prérogative masculine, arrimaient les femmes de cette élite à la sphère domestique, dont elles devenaient les hôtes indésirables, marginalisées et maltraitées, lorsqu'elles ne produisaient pas de fils ou que leurs époux mouraient, faisant d'elles des veuves. 
3 Soutenue par les colonisateurs et les missionnaires britanniques, une petite frange de l'élite éclairée veut réformer ces pratiques, et par conséquent, arracher le droit privé à la juridiction indigène de la caste.

4 Les tenants de l'orthodoxie brahmanique s'y opposent, refusent tout changement social, arguent que la reine Victoria s'était engagée à laisser aux communautés religieuses la gestion de ce droit privé (mariage, héritage, relations inter castes) et dénoncent cette intrusion coloniale. Les femmes ne sont-elles pas depuis des temps immé- moriaux le sanctuaire de la tradition et les garantes de l'honneur familial ? Pourtant le programme de la réforme, exposé au chapitre V, est mesuré : il fixe l'âge du consentement au mariage à 12 ans et permet aux veuves de se remarier. Des lois entérineront ces mesures. Quant à l'éducation des femmes, dont les orthodoxes pensent qu'elle "désexualise leur nature ${ }^{3}$ " elle dépend d'initiatives privées. Là encore, la prudence est de mise : l'accès à l'écriture et à la lecture ne doit pas détourner filles et épouses de leurs devoirs ancestraux.

5 Chaque chapitre de l'ouvrage permet d'approfondir les motivations et les enjeux fondamentaux des forces en présence, coloniales, progressistes et conservatrices, à travers le relais d'associations dynamiques, d'une presse foisonnante et divisée, ou encore d'événements juridiques comme le retentissant procès de la jeune Rakhmabai. Mais surtout, chacun d'eux montre de manière nuancée, précise, passionnante, comment les femmes agissent ou réagissent face à cet espace d'émancipation limité, traversé de contradictions et difficilement praticable dont elles sont les destinataires. Les variations sont extrêmes. Comparons brièvement d'eux d'entre elles, Pandita Ramabai (1858-1922) et Ramabai Ranade (1862-1924).

6 La première, mère, veuve et jamais remariée, est une figure exceptionnellement brillante, radicale et indépendante qui voue son existence à la cause des femmes. Son érudition et sa critique sociale lui font occuper une place éminente dans l'intelligentsia indienne et internationale. Convertie au christianisme (ce qui lui vaudra d'être ostracisée par la société de Pune), elle n'en est pas pour autant une chrétienne malléable ni une adepte naïve de la «mission civilisatrice » britannique. Pendant plusieurs années, en Angleterre puis aux USA, elle s'instruit et enseigne, donne des conférences sur l'Inde et les femmes des hautes castes, collecte de l'argent pour fonder en 1889 le Sharada Sadan, premier établissement éducatif pour les femmes.

7 La seconde est par contraste une femme conventionnelle, convaincue de l'idéal féminin hindou traditionnel de la «femme parfaite ». Épouse du célèbre réformateur M. G. Ranade, elle est l'objet involontaire et la victime, au début de sa vie, des convictions de celui-ci. Il l'abandonne en effet à la torture de deux injonctions contradictoires - il veut l'éduquer mais les femmes de la famille élargie dans laquelle elle vit s'opposent à ce projet - qu'elle ne peut évidemment pas satisfaire. Ramabai parviendra à surmonter les obstacles familiaux et sociaux en dépit du peu d'empathie que lui témoigne son mentor. Elle inventera avec lui une forme de conjugalité, de compagnonnage, incontestablement audacieuse pour l'époque. Après la mort de celui-ci, cette femme soumise mais complice, qui voue une véritable dévotion à son époux, honore sa mémoire et poursuit son œuvre. Elle écrit les Souvenirs de leur vie commune et s'implique dans le travail social: elle fonde un Foyer pour femmes, le Seva Sadan, s'affilie à la première association nationale de femmes (All India Women's Conference), milite au sein du mouvement en faveur de leur droit de vote... Éduquée sans avoir trahi 
l'idéal féminin hindou, Ramabai sera érigée en modèle pour la nation à venir. Alors que Pandita Ramabai, la chrétienne, la rebelle, disparaîtra de l'histoire sociale du Maharashtra, jusqu'à ce que Meera Kosambi la sorte de l'oubli à la fin des années 1980.

8 Cette histoire régionale s'adosse à une longue fréquentation de la vie publique maharashtrienne et à une lecture serrée, attentive de la littérature féminine de l'époque : biographies, autobiographies, correspondances, romans, discours publics... en marathi et en anglais. Leurs auteures, Ramabai Ranade, Yashodabai Joshi, Kashinai Kanikar, Baya Karve, Parvatibai Athavale, Lakshmibai Tilak et Pandita Ramabai sont issues ou proches de ce milieu brahmanique réformateur, courageux mais trébuchant. Elles occupent tour à tour rôles principaux ou secondaires selon les thèmes des onze chapitres de l'ouvrage, de telle sorte qu'au fil des pages, au fil du temps, notre familiarité avec elles s'accroît. Elles deviennent insensiblement de vieilles connaissances, d'autant que l'on dispose de leurs portraits photographiques.

9 Ce sentiment de familiarité s'impose aussi parce que Meera Kosambi, qui connaît la culture maharashtrienne de l'intérieur en plus d'en être l'historienne, prend le soin d'en donner les clés. Par exemple, le chapitre IV, «Walking on the Edge : Female Body and Self ", décrit les conventions vestimentaires régionales de l'époque, les éléments et les coiffures qui les composent, les couleurs codées qui leur sont affectées. Le costume masculin est sensiblement identique tout au long de la vie. Alors que de la tête aux pieds, l'apparence d'une femme reflète les étapes qu'elle traverse et leurs interruptions (stérilité, veuvage). Cette description satisfait bien plus qu'une curiosité muséographique. Grâce à elle, la rupture qu'accomplissent Ramabai Ranade et Parvatibai Athavale par rapport à la coutume revêt toute son ampleur. En dépit de leur veuvage et des pressions sociales, toutes deux refusent d'être "rituellement défigurées ", c'est-à-dire d'abandonner leur chevelure au rasoir douloureux et humiliant du barbier. Au chapitre III, "Home as universe ", le lecteur est invité à pénétrer un wada, cette maison traditionnelle plus ou moins somptueuse de l'élite de Pune. La distribution et l'affectation rigoureuses des espaces masculins et féminins en font le précipité architectural d'un projet social surdéterminé par des principes de pureté rituelle qui conduisent à une vertigineuse ségrégation des sexes. Car dans ces pages, le wada n'est pas qu'un concept, c'est aussi un lieu habité, et donc d'étonnants usages. Ainsi, Yashodabai Joshi raconte que son mari lui adressa la parole une seule fois - pour lui demander de faire tinter plus discrètement ses lourds bracelets de pieds lorsqu'elle marchait - au cours des quatre premières années de leur vie conjugale qui commença pour elle à 10 ans. Elle lui servait pourtant chaque jour ses repas, mais elle observe que tout échange de parole entre époux étant considéré comme «totalement irrespectueux, aucune femme n'osait violer cette discipline dans la maison de son mari $»^{5}$.

10 Le titre de l'ouvrage, "franchir les seuils», développé dans l'introduction, est particulièrement suggestif. Le seuil constitue en effet une figure spatiale et mentale de la ségrégation et de l'enfermement, mais aussi du dépassement et de la transgression.

11 Toutes ces femmes ont traversé très jeunes, à l'intérieur des murs de la maison familiale puis maritale, l'épreuve de la violence morale, physique et sexuelle et à peu près toutes ont connu le tourment d'être à la fois encouragées et critiquées, mises en mouvement et arrêtées, sauvées et abandonnées par le vent nouveau de la réforme sociale ${ }^{6}$. En dépit de ces souffrances, toutes déploient cependant une confondante énergie et une admirable ténacité pour être dignes de leurs mentors (Ramabai Ranade, 
Baya Karve, Kashibai Kanitkar, etc.) ; certaines s'exilent pour devenir médecins (comme A. Joshee et Rakhmabai) et pouvoir fonder des institutions (Pandita Ramabai) ; d'autres y collaborent (Baya Karve, Parvatibai Athavale) ; toutes, enfin ont écrit, pour témoigner de leur expérience, répandre leurs idées, parfois par le biais de la fiction. Leur programme de réformes sociales est sans conteste plus audacieux que celui des hommes. Par exemple, dans le royaume utopique de Kashibai Kanitkar (1861-1948), les femmes sont éduquées, occupent un emploi et sont égales aux hommes ${ }^{7}$. Cependant, Pandita Ramabai est seule à vivre à l'aune de telles attentes. Comme l'écrit Meera Kosambi, le féminisme maharashtrien du xIX siècle ne constitue certainement pas un mouvement, bien que ces femmes interagissent entre elles : il se présente globalement sous une forme « diffuse, fragmentaire et rudimentaire ».

Il ne s'agit pas pour autant dans cet ouvrage d'une histoire régionaliste, le Maharashtra n'apparaît pas comme une forteresse incomparable et isolée. Ni au plan des contenus ni au plan de l'échafaudage sur lequel ils reposent. D'une part, la culture sanskrite de cette élite brahmanique se fonde sur des textes, des valeurs et des règles auxquels toutes les femmes indiennes de hautes castes sont soumises. D'autre part, les affrontements entre colonisateurs, conservateurs et libéraux, renvoient à d'autres configurations régionales, comme leur progressive polarisation en deux fronts, colonisateur et nationaliste. Enfin, le cadre méthodologique et théorique de Crossing Threshholds n'ignore rien des notions fondamentales du féminisme historique, réajustées, amplifiées ou complexifiées à la lumière des matériaux étudiés. Meera Kosambi encourage à moins de naïveté (l'écriture féminine n'est pas systématiquement associée à la « visibilité » des femmes ou à leur autonomie) et à plus de complexité (« le patriarcat » se conjugue au pluriel, tant ses fondements donnent lieu à des modalités différentes). Elle montre l'importance de la longue durée dans le réexamen des sphères privées et publiques et la nécessité de mettre en regard espace social et matériel pour saisir leurs transformations. Elle apporte une preuve supplémentaire au fait que pour comprendre la société indienne, l'examen des rapports de genre ne saurait exclure les considérations de caste et de classe qui s'y mêlent étroitement. Elle revisite les dogmes : dans le sillage de G. Spivak ${ }^{8}$ et de la thèse du «trope du mutisme" dont le subalternisme féminin serait l'acmé, elle oppose le paradoxe qu'incarnent «ses » six femmes. Subalternes, dominées par les hommes en vertu de leur sexe, elles représentent cependant l'élite sociale et intellectuelle de par leur statut et leur prise de parole. Bref, cette historienne féministe démontre que le féminisme n'est pas une idéologie mais un exercice critique, d'autant plus stimulant et convaincant qu'il est porté par une langue élégante.

13 Crossing Threshholds rassemble des articles publiés depuis les années 1990 et dispersés dans les revues qui «comptent » en Inde. Notons parmi elles, la « Review of Women Studies $"^{9}$ dans Economic and Political Weekly ou Contributions to Indian Sociology, auxquelles les noms de Alice Thorner et Louis Dumont sont indissociablement attachés, Feminist Review, History of Education, etc. D'autres articles sont publiés dans des ouvrages collectifs, dont certains sont aujourd'hui des classiques des études maharashtriennes (comme House and Home in Maharashtra, édité par Irina Glushokova et Anne Feldhaus en 1998); d'autres encore témoignent de la carrière internationale de l'auteure qui enseigna et vécut en Suède, aux États-Unis et en Australie : ainsi l'article sur Anandibai Joshee, première femme médecin du Maharashtra, fut publié en 2001 dans Australian Feminist Studies. L'introduction «Multiple Threshholds» est inédite ainsi que les trois essais suivants : «Walking on the Edge : Female Body and Self » déjà cité, « Feminist 
Utopian Visions : Kashibai Kanitkar's Creative Writing », et « Gender and Nationalism ». Celui-ci conclut la première partie de l'ouvrage, "Home and Beyond "sur la défaite politique des six pionnières. Bien qu'elles aient pénétré la sphère publique, l'exercice du pouvoir leur demeure inaccessible. C'est une prérogative masculine sur laquelle s'accordent toutes les forces, impérialistes, progressistes et conservatrices en présence et que le nationalisme bientôt vainqueur réaffirmera.

Je ne suis pas totalement ignorante de l'histoire des femmes indiennes ${ }^{10}$. Cet ouvrage m'a conduit à en avoir une vision moins partisane. Je conclurai sur ce point.

Il me semblait que les "réformateurs » étaient pétris d'intentions louables, mais qu'ils se montraient souvent ambigus, parfois même d'une suffocante lâcheté envers les femmes. Je pensais qu'ils étaient responsables de leur féminisme « rudimentaire ». Or, si Meera Kosambi montre bien que la "race des femmes", comme on dit en marathi, était dressée, souvent dans la terreur, à servir, deviner les désirs de leurs maitres et à se taire, elle incite aussi, par l'accumulation des récits et des témoignages, à renverser les perspectives, à réfléchir au dressage des hommes. Dressés à se faire servir sans discussion par l'autre et à la considérer comme impure et faible; dressés à la répudier si elle n'enfantait pas de fils, dressé au concubinage, dressé à se remarier dans le veuvage. Dressés à faire de cette autre l'exutoire de ses frustrations, bref, à exercer impunément droit de vie et de mort sur elles. En tout état de cause, la "race » des hommes était constituée de telle manière qu'elle ne pouvait pas se mettre à la place de celle des femmes.

Dans ces conditions extrêmes et si confortables pour eux, il est étonnant que certains aient conçu un autre ordre des choses. Comme il est étonnant que des femmes, certes éduquées, mais toujours aussi dépendantes, vulnérables et rabrouées, aient soutenu ce nouvel ordre des choses.

18 Maints passages de Crossing Threshholds attestent que le patriarcat brahmanique menacé pouvait se montrer impitoyable. Les familles se divisaient, les membres déviants étaient ostracisés de leurs castes, éventuellement assassinés ${ }^{11}$. La fenêtre qui s'ouvrait était hérissée de risques et l'avenir bien incertain pour les uns et les autres.

19 Et pourtant condamnées à s'ignorer en dépit d'une sexualité partagée, les deux races tentèrent de se rapprocher et d'infléchir leurs sacro-saints rapports de pouvoir vers plus de justice et d'égalité, alors même que les coutumes étaient engagées au cours de ce siècle colonial dans une spirale d'excès. L'élite réinventa cependant son quotidien. Je pense aujourd'hui qu'il s'agit d'un quasi-miracle et que les ambiguités masculines ne sont pas simplement la cause, mais le symétrique inverse de la prudence féminine.

\section{NOTES}

1. C'est la première et la plus importante université pour femmes de l'Inde. À son origine, c'est le Foyer pour veuves de D. K. Karve, fondé en 1896. Il deviendra un Collège pour femmes puis une université. Voir ici même note 5, à propos de D. K. Karve et son épouse Baya. 
2. Voir en particulier les chapitres III ("Home as Universe ») et IV ("Walking on the Edge: Female Body and Self ») sur cet aspect normatif de la société maharashtrienne.

3. The Mahratta, 26 juillet 1891 (cité p. 144).

4. Voir les pages 105-107.

5. Chapitre III, p. 109.

6. L'histoire de Baya Karve (1866-1950) qui conclut la seconde partie de l'ouvrage (« Mariage, maternité, veuvage ») en est un exemple extraordinaire. Veuve très jeune, son second mariage est arrangé lorsqu'elle a 27 ans par son père (qui n'assiste pas à la cérémonie par peur du boycott social) avec un jeune et audacieux réformateur, D. K. Karve. Ce professeur de mathématiques consacre son temps et son argent à des œuvres éducatives en faveur des femmes. Succombant finalement à son tour à la pression sociale, il en écarte son épouse, au prétexte que son statut de «veuve remariée » est un obstacle au succès de ses entreprises. Dans les années 1920, l'illustre époux encourage Baya à vendre son autobiographie pour rembourser son éditeur. Elle accomplit un périple au Maharashtra, puis entreprend un tour solitaire de l'Inde auprès des familles maharashtriennes brahmanes afin de collecter des fonds. Meera Kosambi se souvient avoir vu, lorsqu'elle était enfant, la silhouette cassée de Baya qui jusqu'au terme de sa vie parcourait Pune dans ce dessein. À la fois redevable et victime de son époux, elle ne remit jamais en question son allégeance à son égard.

7. Chapitre VI : «Feminist Utopian Visions : Kashibai Kanitkar's Creative Writing » (p. 173).

8. Il est question ici de son célèbre article "Can the Subaltern Speak?», in Patrick Williams \& Laura Chrisman, eds, Colonial Discourse and Postcolonial Theory. A Reader, New York, Harvester Wheatleaf, 1994 : 66-111.

9. Voir pour l'histoire de la création de ce supplément biannuel de Economic and Political Weekly, le récit d'Alice Thorner dans la préface, pp. V-XI, de Ideals, Images and Real Lives, éd. par Alice Thorner \& Maitreyi Krishnaraj (Delhi, Orient Longman, 2000). L'initiative vient des deux éditrices de cet ouvrage dans lequel collabore notamment Meera Kosambi.

10. J'ai écrit plusieurs articles sur cette question et prépare un ouvrage sur les « résistances féminines en Inde du XIX ${ }^{\mathrm{e}}$ au XxI ${ }^{\mathrm{e}}$ siècle » qui doit paraître chez Albin Michel fin 2008.

11. Comme en témoigne le "suicide " très contesté de Moroba Kanhoba Vijaykar et de son épouse (ex- veuve). À peine un an après leur mariage, le couple excommunié par sa caste fut retrouvé noyé dans un puits, leurs cous attachés par une corde (voir p. 350). 